\title{
Maternal depression during pregnancy and cord blood DNA methylation: findings from the Avon Longitudinal Study of Parents and Children
}

\author{
A. C. Viuff(1) ', G. C. Sharp $\mathbb{1}^{2,3}$, D. Rai ${ }^{4,5}$, T. B. Henriksen ${ }^{1,6}$, L. H. Pedersen ${ }^{1,7}$, K. J. Kyng ${ }^{6}$, N. H. Staunstrup ${ }^{8,9}$, A. Cortes ${ }^{10}$, \\ A. Neumann $\mathbb{D}^{10}$, J. F. Felix ${ }^{11,12,13}$, H. Tiemeier ${ }^{10,11}$, V. W. V. Jaddoe ${ }^{11,12,13}$ and C. L. Relton 2,4
}

\begin{abstract}
Up to $13 \%$ of women may experience symptoms of depression during pregnancy or in the postpartum period. Depression during pregnancy has been associated with an increased risk of adverse neurodevelopmental outcomes in the child and epigenetic mechanisms could be one of the biological pathways to explain this association. In 844 mother-child pairs from the Avon Longitudinal Study of Parents and Children, we carried out an epigenome-wide association study (EWAS) to investigate associations between prospectively collected data on maternal depression ascertained by the Edinburgh Postnatal Depression Scale in pregnancy and DNA methylation in the cord blood of newborn offspring. In individual site analysis, we identified two CpG sites associated with maternal depression in the middle part of pregnancy. In our regional analysis, we identified 39 differentially methylated regions (DMRs). Seven DMRs were associated with depression at any time point during pregnancy, 7 associated with depression in midpregnancy, 23 were associated with depression in late pregnancy, and 2 DMRs were associated with depression throughout pregnancy. Several of these map to genes associated with psychiatric disease and brain development. We attempted replication in The Generation R Study and could not replicate our results. Although our findings in ALSPAC suggest that maternal depression could be associated with cord blood DNA methylation the results should be viewed as preliminary and hypothesis generating until further replicated in a larger sample.
\end{abstract}

\section{Introduction}

Up to $13 \%$ of women experience symptoms of depression during pregnancy or postpartum ${ }^{1,2}$. Depression during pregnancy has been associated with an increased risk of adverse neurodevelopmental outcomes in the offspring $^{3-5}$. Children of mothers with depression during pregnancy have also been shown to be more likely to develop a psychiatric disorder later in life ${ }^{6-8}$. The biological mechanisms behind the association between

\footnotetext{
Correspondence: A. C. Viuff (acviuff@clin.au.dk)

${ }^{1}$ Perinatal Epidemiology Research Unit, Department of Clinical Medicine, Health, Aarhus University, Aarhus, Denmark

${ }^{2} \mathrm{MRC}$ Integrative Epidemiology Unit, University of Bristol, Bristol, UK

Full list of author information is available at the end of the article.
}

prenatal maternal depression and predisposition to later behavioural problems, learning difficulties, and psychiatric illness in the offspring have been extensively discussed, and it has been hypothesised, that epigenetic pathways could be one of the biological pathways involved ${ }^{9}$. Epigenetics is the study of potentially heritable molecular modifications to DNA and histone proteins that can affect gene expression without change to the underlying DNA sequence ${ }^{10}$. The most frequently studied epigenetic phenomenon is DNA methylation where $\mathrm{CpG}$ dinucleotides undergo a process of cytosine methylation which can alter chromatin accessibility and thereby gene transcription ${ }^{10}$. 
Timing of exposure to maternal depression may be important in understanding the causal relationship. Human studies suggest that the second and early third trimesters are the more sensitive periods when exposures to stressful life events increase the risk of offspring illness ${ }^{11}$, although the evidence is inconsistent ${ }^{12}$. Studies investigating candidate genes (NR3C1 and SLC6A4) in relation to maternal depression during pregnancy have suggested trimester-specific differences in methylation patterns in the cord blood of the offspring ${ }^{13,14}$.

The aim of this study was to test the hypothesis that prenatal maternal depression would be associated with epigenome-wide differences in methylation in the cord blood of newborns in a large birth cohort study.

\section{Methods}

Participants

The Avon Longitudinal Study of Parents and Children (ALSPAC) recruited 14,541 pregnant women resident in Avon, UK with an expected delivery date falling between 1 April 1991 and 31 December 1992 (ref. ${ }^{15}$ ). During pregnancy the included women were sent up to four selfcompletion postal questionnaires. At least one questionnaire was completed by 14,119 women and 11,545 women completed all four questionnaires ${ }^{16}$. These questionnaires included questions on lifestyle, mental health, diet, medication use, education, occupation, ethnicity, alcohol, and tobacco use. Two of the questionnaires administered around the 18th and the 32nd week of pregnancy contained questions concerning depression. The study website contains details of all data that is available through a fully searchable data dictionary ${ }^{17}$ (http://www.bristol.ac.uk/alspac/researchers/). Ethical approval for the study was obtained from the ALSPAC Ethics and Law Committee and the Local Research Ethics Committees.

We included women with singleton pregnancies where the woman had completed both questionnaires containing questions on depression during pregnancy and had a child with cord blood DNA methylation data. ALSPAC includes very few pregnancies of non-Caucasian ethnicity, so we excluded those from the study (Fig. 1).

\section{Measure of maternal depression}

The postal questionnaires at 18 and 32 weeks of pregnancy included the 10-item Edinburgh Postnatal Depression Score (EPDS) ${ }^{18}$. The EPDS is a validated questionnaire to screen women for depression postpartum as well as during pregnancy ${ }^{19}$. Scores of $\geq 12$ have a high correlation to clinically diagnosed depressive disorders $^{20}$. A total of 844 women had data on EPDS scores and offspring cord blood DNA methylation (Fig. 1).

In our primary analysis we investigated whether screening positive for depression $(E P D S \geq 12)$ at either

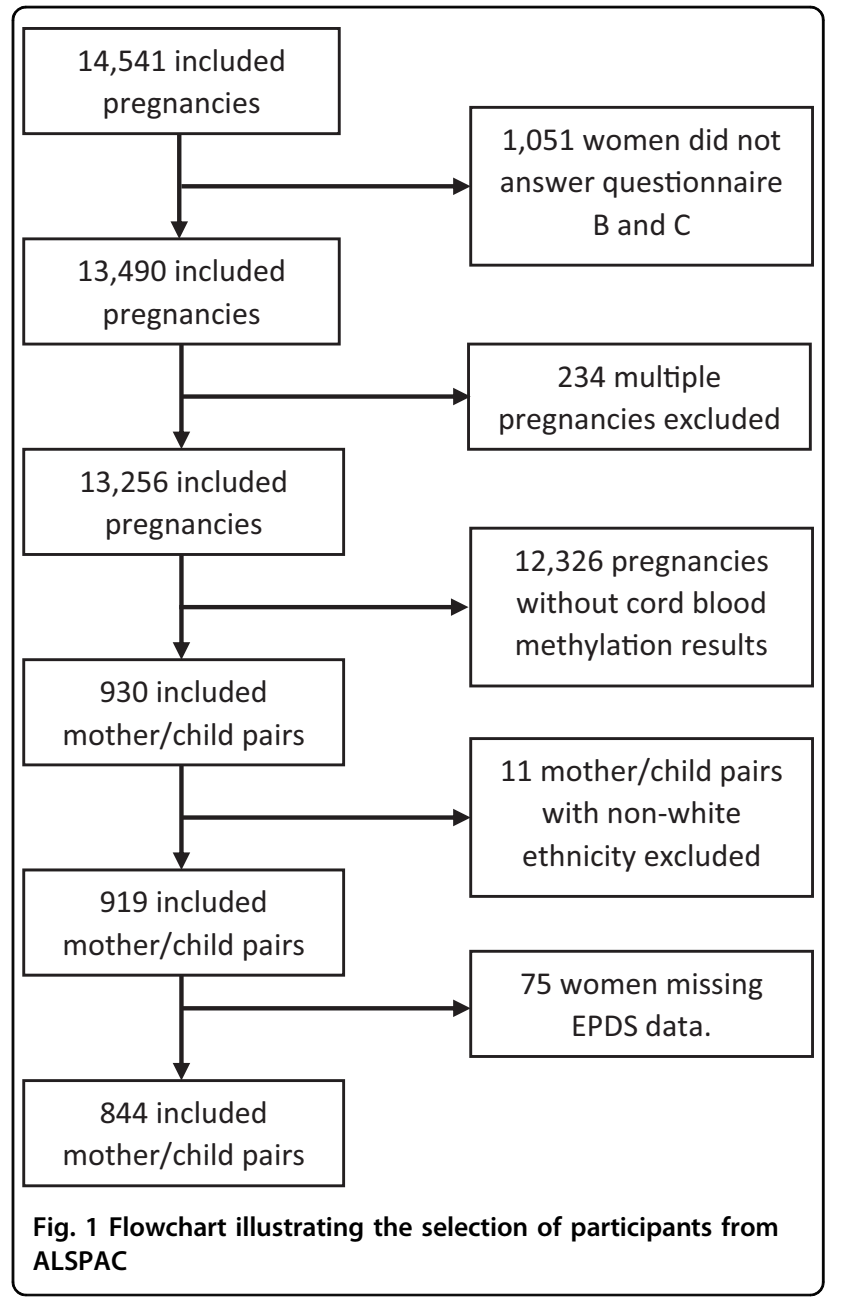

time point during pregnancy was associated with differential cord blood DNA methylation. Therefore, offspring of women who had an EPDS $\geq 12$ at either week 18 or week 32 were considered exposed and these women will be referred to as depressed throughout this paper. We did three secondary analyses investigating the methylation differences in children of mothers with depression in midpregnancy only (EPDS $\geq 12$ at week 18 and $\leq 12$ at week 32 ), late in pregnancy only (EPDS $\geq 2$ at week 32 and $\leq 12$ at week 18), and at both time points in pregnancy (EPDS $\geq 12$ at both weeks 18 and 32). The reference was offspring of women with EPDS $<12$ at both week 18 and week 32 .

\section{Methylation data}

The procedure for collecting and storing cord blood samples is described elsewhere ${ }^{21}$. The DNA methylation analysis and the preprocessing of data were performed at the University of Bristol as a part of the ARIES project ${ }^{22}$. The ARIES project holds DNA methylation data on just under 1000 cord blood samples. DNA methylation was 
measured in cord blood samples using the Illumina Infinium HumanMethylation 450K BeadChip assay ${ }^{23}$. This platform analyses the methylation level of over 485,000 CpG sites in one array using bisulfite converted genomic DNA. The level of methylation is reported as a beta-value ( $\beta$-value) ranging from 0 (completely unmethylated) to 1 (completely methylated).

Data were pre-processed in $\mathrm{R}$ (version 3.0.1) using the WateRmelon package ${ }^{24}$ and functional normalisation was performed using the Meffil package in $\mathrm{R}^{25}$ as an attempt to reduce non-biological differences between probes. We removed 11,607 probes mapping to the $\mathrm{Y}$ or $\mathrm{X}$ chromosomes or because they were SNPs included on the array for control purposes. In all, 2626 probes with a detection $p$-value $>0.05$ were also removed. We identified extreme outliers in the methylation data using the Tukey method $^{26}$. This method uses the interquartile range (IQR) and extreme outliers are defined as values outside first quartile $-3 \times I Q R$ and third quartile $+3 \times I Q R$. These probes were set as missing in the dataset.

\section{Covariates}

We adjusted for potential confounders, which we selected with the help of Directed Acyclic Graphs (DAGs) ${ }^{27}$. The potential confounders were maternal education, parity, smoking, maternal pre-pregnancy BMI, and maternal age at delivery. We also adjusted for estimated cell proportions in the cord blood samples and technical batch. Maternal education was defined as having achieved below A-levels or A-levels and above. Parity was defined as no children or $\geq 1$ child. Smoking was defined as any smoking during pregnancy or no smoking during pregnancy. Maternal age was included as a continuous variable. Cell proportions were estimated using the method developed by Houseman et al $^{28}$ and the cord blood reference panel published by Bakulski et al. ${ }^{29}$. We adjusted for technical batch using surrogate variable analysis (SVA ${ }^{30}$. Using the sva package in R we estimated 10 surrogate variables which are covariates constructed from the data to adjust for unknown and un-modelled factors associated with the technical batch. Those surrogate variables not associated with the trait of interest are included as covariates in the regression analysis.

\section{Statistical analysis}

\section{Single site regression analyses}

Linear regression was applied to investigate the association between maternal depression and methylation changes in the cord blood of the newborn. These EWASs were conducted in $\mathrm{R}$ version 3.0.2.

In our main analysis, DNA methylation (untransformed $\beta$-values) was the outcome. We ran four models to interrogate associations between depression at any time during pregnancy and DNA methylation: (1) adjusted for batch; (2) adjusted for batch and estimated cell proportion types; (3) adjusted for batch and covariates: maternal age, maternal pre-pregnancy BMI, parity, smoking, and maternal education; (4) adjusted for batch, covariates, and cell proportion types.

In secondary analyses, we applied the same four models, defining our exposure as depression in mid-pregnancy only, late pregnancy only, and depression at both time points.

$p$-Values were adjusted for genome-wide significance using false discovery rate (FDR) adjustment and only sites with FDR-corrected $p$-values $<0.05$ were considered statistically significant.

\section{Regional analyses}

When multiple adjacent $\mathrm{CpG}$ sites are differentially methylated, it is referred to as a differentially methylated region $(\mathrm{DMR})^{31}$. DMRs may be more biologically important than differential methylation of individual, isolated CpGs, and region-based approaches are statistically more powerful with a lower rate of false positive findings compared to EWAS at individual CpGs. Therefore, in addition to the single-site analyses, we also performed regional analyses using Comb- $\mathrm{P}^{32}$. This method combines adjacent $p$-values from the single CpG analysis, corrects for correlation with neighbouring $\mathrm{CpGs}$ within 1000 bp applying the Stouffer-Liptak method, and adjusts for multiple testing using a Sidak correction ${ }^{33}$.

For each exposure of interest (depression at any time, mid-pregnancy, late pregnancy, and at both time points), we performed regional analyses on the results of the single-site analyses obtained using the fully adjusted model (model 4).

\section{Enrichment analysis}

We used the missMethyl R package ${ }^{34}$ to perform gene set enrichment analysis within the DMRs associated with depression in each of the four exposures of interest. This considers the differing number of sites associated with each gene on the Illumina $450 \mathrm{~K}$ array. We tested for enrichment of any Gene Ontology $(\mathrm{GO})^{35}$ classification term and Kyoto Encyclopaedia of Genes and Genomes (KEGG) pathways ${ }^{36} \cdot p$-Values were adjusted for multiple testing using the FDR method. Only terms with FDR-corrected $p$-values $<0.05$ were considered significant.

\section{Replication}

We used data from the Generation R Study ${ }^{37}$ based in Rotterdam, the Netherlands for replication of our initial observations in ALSPAC. The Generation R Study is a population-based prospective cohort study from foetal life onwards. It includes 9778 women and their children, born between April 2002 and January 2006. The study has been 
approved by Medical Ethical Committee of Erasmus MC, University Medical Center Rotterdam, and written consent was obtained for all participants.

The Generation R Study Biobank has DNA methylation results from 1339 cord blood samples measured using the Illumina $450 \mathrm{~K}$ Infinium BeadChip ${ }^{38}$. The depression subscale of the Brief Symptom Inventory (BSI) ${ }^{39}$ was completed at 20 weeks of pregnancy. This timing corresponds with the EPDS score from week 18 in ALSPAC. The BSI is a 53-item version of the Symptom Checklist-90 (SCL-90) that measures emotional-behavioural functioning. Information about maternal education, parity, and smoking during pregnancy was collected by self-reported questionnaires during pregnancy. Maternal education was classified in two levels: A-level or above (post-age 16 or above) and below A-level (education level of age 16). Parity was defined as no children or $\geq 1$ child, and smoking during pregnancy as no smoking in pregnancy or any smoking during pregnancy. Pre-pregnancy BMI was calculated based on maternal pre-pregnancy weight, obtained by self-reported questionnaires, and maternal height measured at enrolment. Maternal age at delivery was calculated based on the date of birth of the mother and the child (Table S1).

A single-site regression analysis of the Generation $R$ study methylation data was performed as well as a regional analysis as described above using the fully adjusted model. Cell proportions were adjusted for using the Houseman method with the reference panel for cord blood published by Bakulski et al. ${ }^{29}$. Plate ID $(n=27)$ was included in models as a covariate to adjust for technical batch. We used these results to do a look up of the results from our single site analysis and our DMR analysis in ALSPAC.

\section{Methylation quantative trait loci analysis}

When performing EWAS studies it must be considered that the methylation variation may partly be explained by underlying genetic variation. DNA sequences that are associated with patterns in DNA methylation are referred to as methylation quantative trait loci (mQTLs) ${ }^{40}$. These can act either in cis (within $1 \mathrm{MB}$ either side of the $\mathrm{CpG}$ probe) or trans (further than $1 \mathrm{MB}$ from the CpG probe).

We looked up any maternal depression-associated $\mathrm{CpG}$ sites in a database of mQTLs (http://www.mqtldb.org/) ${ }^{41}$ previously identified in the cord blood of 771 ALSPAC children ${ }^{41}$. mQTLs were included in the database if the association between the SNP and the CpG had a $p$-value $<1 \times 10^{-7}$.

\section{Results}

We had complete data on exposure to maternal depression during pregnancy and methylation changes in the cord blood of the offspring in 844 mother-child pairs in ALSPAC. Of these women 204 screened positive for depression at any time point in pregnancy: 53 women were depressed only in mid-pregnancy, 85 women only in late pregnancy, and 66 women at both time points. Our reference group consisted of 644 women classified as not depressed at any point during pregnancy.

More women with depression reported smoking during pregnancy than women in the reference group. Women in the reference group had a higher level of education than women with depression. The groups were comparable regarding other characteristics (Table 1).

\section{Single site regression analyses}

In our main analysis (depression at any time point in pregnancy), we found no significantly differentially

Table 1 Characteristics of the included women

\begin{tabular}{|c|c|c|c|c|c|}
\hline$n=844$ & $\begin{array}{l}\text { Depression any time in } \\
\text { pregnancy }(n=204) \\
(E P D S \geq 12)\end{array}$ & $\begin{array}{l}\text { Depression in mid- } \\
\text { pregnancy }^{\mathrm{a}}(n=53)\end{array}$ & $\begin{array}{l}\text { Depression in late } \\
\text { pregnancy }^{b}(n=85)\end{array}$ & 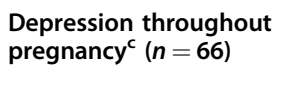 & $\begin{array}{l}\text { No depression in } \\
\text { pregnancy }(n=644) \\
\text { (EPDS }<12)\end{array}$ \\
\hline $\begin{array}{l}\text { Age (years) } \\
(\text { mean }(95 \% \mathrm{Cl}))\end{array}$ & $29.4(28.7 ; 30)$ & $29.4(28.1 ; 30.7)$ & $29.7(28.6 ; 30.8)$ & $28.8(27.6 ; 30.1)$ & $29.7(29.4 ; 30.1)$ \\
\hline $\begin{array}{l}\text { BMI (mean } \\
((95 \% \mathrm{Cl}))\end{array}$ & $22.7(22.2 ; 23.2)$ & $23.2(22.2 ; 24.2)$ & $22.1(21.4 ; 22.8)$ & $23.1(22 ; 24.2)$ & $22.9(22.6 ; 23.2)$ \\
\hline \multicolumn{6}{|l|}{ Parity (n) } \\
\hline No children & $82(41 \%)$ & $23(44 \%)$ & $29(35 \%)$ & $30(45 \%)$ & $314(49 \%)$ \\
\hline$\geq 1$ child & $116(56 \%)$ & $28(53 \%)$ & $53(62 \%)$ & $34(52 \%)$ & $312(48 \%)$ \\
\hline \multicolumn{6}{|c|}{ Smoking in pregnancy $(n)$} \\
\hline No smoking & $150(74 \%)$ & $39(74 \%)$ & 68 (80\%) & $43(65 \%)$ & 584 (91\%) \\
\hline Any smoking & $52(25 \%)$ & $14(26 \%)$ & $15(18 \%)$ & $23(35 \%)$ & $60(9 \%)$ \\
\hline \multicolumn{6}{|l|}{ Education Level } \\
\hline O-levels/GCSE & 117 (57\%) & $26(49 \%)$ & 47 (55\%) & $44(67 \%)$ & 312 (49\%) \\
\hline $\begin{array}{l}\text { A-levels and } \\
\text { above }\end{array}$ & $85(42 \%)$ & $25(47 \%)$ & $38(45 \%)$ & $22(33 \%)$ & $331(51 \%)$ \\
\hline
\end{tabular}

${ }^{a}$ EPDS $\geq 12$ at 18 weeks only ${ }^{b}$ EPDS $\geq 12$ at 32 weeks only ${ }^{c}$ EPDS $\geq 12$ at 18 and 32 weeks 


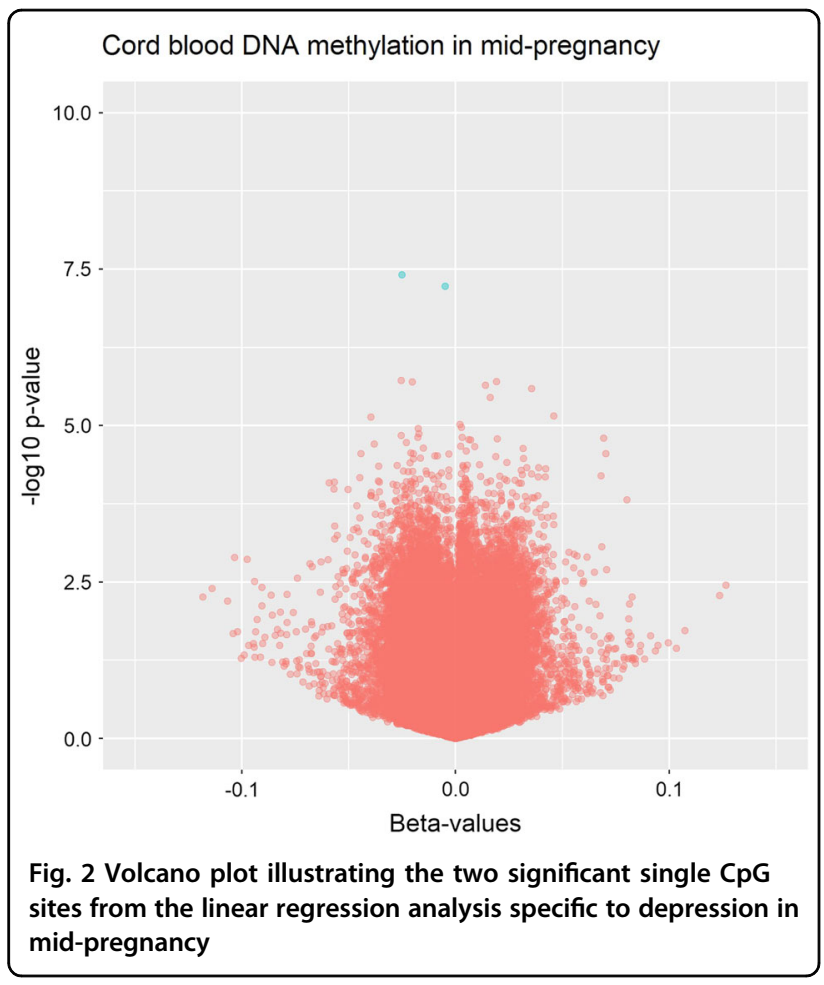

methylated single CpG sites (FDR-corrected $p$-value $<0.05)$ in any of the four models.

When investigating depression in mid-pregnancy (up to week 18), we identified two CpGs with FDR-corrected $p$ values $<0.05$ (Fig.2). Methylation of cg08667740, located on a CpG shore in the body of the CYBA gene, was $2.5 \%$ $\left(\beta=-0.025 ; p=3.9 \times 10^{-8}\right)$ lower in the umbilical cord blood of children exposed to maternal depression in midpregnancy, relative to unexposed children. Methylation of cg22868225, in the body of the PRKCZ gene, was $0.5 \%$ lower $\left(\beta=-0.005 ; p=5.89 \times 10^{-8}\right)$. This association was present in all four models. There were no CpGs with FDR-corrected $p$-value $<0.05$ in the single-site EWAS of depression in late pregnancy or depression throughout pregnancy in any of the four models.

\section{Regional analysis}

In regional analyses we identified 39 differentially methylated regions across all four depression exposures using the fully adjusted model (Sidak-corrected $p<0.05$ ). Seven DMRs were associated with depression at any time point during pregnancy, 7 associated with depression in mid-pregnancy, 23 were associated with depression in late pregnancy, and 2 DMRs were associated with depression throughout pregnancy (Table 2).

Three DMRs (mapping to HFMI, CARTPT, and $R A S I P 1 K$ ) were common to two exposures: depression at any time point during pregnancy and depression in late pregnancy only. These two analyses also identified DMRs in close proximity to each other, but not identical. These DMRs both mapped to the HCG4P6 gene.

The regional analyses in women with depression at any time point during pregnancy and depression throughout pregnancy did not share mutual DMRs. They did however identify neighbouring DMRs that both mapped to the GNAS gene.

No mutual DMRs were identified in the analyses of the cord blood from offspring having been exposed to depression in mid-pregnancy, late, and throughout pregnancy, respectively.

\section{Functional gene enrichment analysis (GO and KEGG analyses)}

Among the DMRs specific to depression throughout pregnancy we found 1 KEGG pathway (related to insulin secretion) but no enriched biological process GO terms $($ FDR $<0.05)$.

\section{Replication}

Our look up of the results from the single site analysis and the regional analysis in ALSPAC did not replicate in the Generation R sample.

Of the two CpG sites (cg08667740 and cg22868225) that were specific to mid-pregnancy maternal depression in ALSPAC, one was associated with depression in Generation $\mathrm{R}$ with the same direction of effect. The other $\mathrm{CpG}$ was associated with depression with the opposite direction of effect. None of them reached statistical significance (Table 3).

In the regional analysis we found no mutual DMRs in the two datasets. Out of the $68 \mathrm{CpGs}$ within the seven DMRs specific to depression in mid-pregnancy in the ALSPAC regional analysis, 49 (72\%) showed the same direction of association in the Generation $\mathrm{R}$ study, but none survived correction for multiple testing (Tables S2, S3).

\section{Methylation quantative trait loci analysis}

Of the two CpGs identified in our single site analysis, cg22868225 was associated with two trans-SNPs located on chromosome 10 (rs17569335, $\beta=0.267, p=7.8 \mathrm{e}-08$ and rs2768469, $\beta=0.276, p=3.41 \mathrm{e}-08)$, and $\operatorname{cg} 08667740$ was associated with one trans-acting SNP ( $\operatorname{rs} 13190610, \beta$ $=-1.352, p=2.04 \mathrm{e}-08$ ) located on chromosome 5 .

Neither CpG was associated with any cis-acting SNPs, suggesting that the observed methylation differences between offspring of depressed and unaffected mothers is not being driven by the underlying genetic architecture.

\section{Discussion}

Maternal depression (occurring at any time point during pregnancy) was associated with offspring cord blood 
Table 2 Results of the regional analysis

\begin{tabular}{|c|c|c|c|c|c|c|}
\hline Exposure group & Gene & Location & No. of CpGs & DMR & Median beta (range) & Sidak-corrected $p$-values \\
\hline \multirow[t]{7}{*}{ Any time point } & RASIP1 & $19 q 13.33$ & 5 & Chr19:49223814-49224166 & $0.02(0.01 ; 0.02)$ & $2.8 e-05$ \\
\hline & HFM1 & $1 p 22.2$ & 6 & Chr1:91852791-91853090 & $-0.01(-0.02 ; 0.0)$ & $6.8 e-04$ \\
\hline & NNAT & $20 q 11.23$ & 23 & Chr20:36148604-36149082 & $-0.01(-0.02 ; 0.00)$ & 7.1e-04 \\
\hline & HCG4P6 & $6 p 22.1$ & 8 & Chr6:29893926-29894163 & $0.04(0.0 ; 0.07)$ & $2.1 e-03$ \\
\hline & GNAS & 20913.32 & 9 & 3Chr20:57427556-57427831 & $0.01(0.0 ; 0.02)$ & $8.0 e-03$ \\
\hline & CARTPT & $5 q 13.2$ & 6 & Chr5:71146767-71146877 & $-0.02(-0.02 ;-0.00)$ & $2.8 e-02$ \\
\hline & $A R L 5 C$ & $17 q 12$ & 3 & Chr17:37322028-37322432 & $0.02(0.01 ; 0.04)$ & $5.3 e-04$ \\
\hline \multirow[t]{7}{*}{ Mid-pregnancy depression } & HOXA5 & $7 p 15.2$ & 42 & Chr7:27183133-27184522 & $-0.02(-0.06 ; 0.00)$ & $8.4 \mathrm{e}-10$ \\
\hline & LYNX1 & $8 q 24.3$ & 7 & Chr8:143859669-143859991 & $0.03(0.02 ; 0.06)$ & $1.2 \mathrm{e}-05$ \\
\hline & SULF1 & $8 q 13.2$ & 8 & Chr8:70378380-70378995 & $0.01(0.00 ; 0.02)$ & $8.0 e-05$ \\
\hline & C6orf227 & $6 \mathrm{p} 21.31$ & 8 & Chr6:33561099-33561450 & $0.01(0.00 ; 0.03)$ & $1.5 \mathrm{e}-04$ \\
\hline & RGS1 & $1 \mathrm{q} 31.2$ & 2 & Chr1:192544716-192544903 & $0.04(0.01 ; 0.07)$ & $3.2 \mathrm{e}-03$ \\
\hline & $B C L 11 B$ & $14 q 32.2$ & 3 & Chr14:99655593-99655748 & $-0.04(-0.06 ;-0.03)$ & $9.2 \mathrm{e}-03$ \\
\hline & SNED1 & $2 q 37.3$ & 2 & Chr2:242031617-242031695 & $0.01(-0.00 ; 0.02)$ & 3.0e-02 \\
\hline \multirow[t]{23}{*}{ Late depression } & CARTPT & $5 q 13.2$ & 6 & Chr5:71146767-71146877 & $-0.03(-0.04 ;-0.02)$ & $6.6 e-08$ \\
\hline & HFM1 & $1 p 22.2$ & 6 & Chr1:91852791-91853090 & $-0.02(-0.03 ;-0.00)$ & $1.7 e-06$ \\
\hline & HCG4P6 & $6 p 22.1$ & 16 & Chr6:29893926-29894342 & $0.02(0.00 ; 0.01)$ & $1.8 e-05$ \\
\hline & PLEKHG1 & $6 q 25.1$ & 3 & Chr6:151125848-151125886 & $0.03(0.03 ; 0.03)$ & $3.0 e-05$ \\
\hline & FAM2OC & $7 p 22.3$ & 5 & Chr7:213469-213849 & $-0.02(-0.04 ; 0.00)$ & $1.0 \mathrm{e}-04$ \\
\hline & DDO & $6 q 21$ & 4 & Chr6:110720918-110721350 & $0.05(0.03 ; 0.06)$ & $1.3 e-04$ \\
\hline & RASIP1 & 19913.33 & 5 & Chr19:49223814-49224166 & $0.02(0.01 ; 0.03)$ & $1.7 e-04$ \\
\hline & HLA-DRB6 & $6 p 21.32$ & 14 & Chr6:32551749-32552247 & $-0.05(-0.10 ;-0.00)$ & $2.5 \mathrm{e}-04$ \\
\hline & JA668105 & $2 q 21.2$ & 3 & Chr2:133025652-133025918 & $-0.01(-0.03 ; 0.03)$ & 5.3e-04 \\
\hline & TRNA_Ala & $6 p 22.1$ & 17 & Chr6:28583971-28584289 & $-0.01(-0.03 ;-0.00)$ & $6.1 \mathrm{e}-04$ \\
\hline & KLHL30 & $2 q 317.3$ & 6 & Chr2:239047029-239047337 & $0.02(0.01 ; 0.04)$ & $1.1 \mathrm{e}-03$ \\
\hline & MUC4 & $3 q 29$ & 7 & Chr3:195489708-195490170 & $0.05(0.02 ; 0.07)$ & $1.1 \mathrm{e}-03$ \\
\hline & JPH3 & $16 \mathrm{q} 24.2$ & 2 & Chr16:87682036-87682143 & $-0.08(-0.01 ;-0.05)$ & $3.5 \mathrm{e}-03$ \\
\hline & PSPH & $7 p 11.2$ & 3 & Chr7:56242181-56242555 & $0.04(0.03 ; 0.04)$ & $5.5 \mathrm{e}-03$ \\
\hline & AK054726 & $1 q 42.2$ & 5 & Chr1:234367145-234367587 & $-0.03(-0.04 ;-0.03)$ & $6.4 \mathrm{e}-03$ \\
\hline & LMO7 & $13 q 22.2$ & 4 & Chr13:76334583-76334867 & $0.02(0.02 ; 0.02)$ & $6.7 e-03$ \\
\hline & ESRRG & $1 q 41$ & 3 & Chr1:217306590-217306764 & $-0.04(-0.04 ;-0.03)$ & $9.0 \mathrm{e}-03$ \\
\hline & SDK1 & $7 p 22.2$ & 3 & Chr7:4244250-4244644 & $-0.06(-0.01 ;-0.05)$ & $1.0 \mathrm{e}-02$ \\
\hline & SLCO5A1 & $8 q 13.3$ & 4 & Chr8:70602451-70602610 & $-0.01(-0.03 ; 0.00)$ & $2.0 e-02$ \\
\hline & IFITM4P & $6 p 22.1$ & 4 & Chr6:29723301-29723408 & $-0.04(-0.06 ;-0.04)$ & $2.9 e-02$ \\
\hline & $D L G 2$ & $11 q 14.1$ & 3 & Chr11:85195094-85195206 & $-0.01(-0.02 ;-0.01)$ & $3.1 \mathrm{e}-02$ \\
\hline & $B I N 1$ & $2 q 14.3$ & 2 & Chr2:127811805-127811854 & $-0.03(-0.04 ;-0.03)$ & $3.2 \mathrm{e}-02$ \\
\hline & $D L X 1$ & $2 q 31.1$ & 2 & Chr2:172948725-172948826 & $0.01(0.01 ; 0.01)$ & $4.8 \mathrm{e}-02$ \\
\hline \multirow[t]{2}{*}{ All } & GNAS & 20913.32 & 17 & Chr20:57427472-57428033 & $0.02(0.00 ; 0.03)$ & $4.1 e-09$ \\
\hline & TRPM4 & $19 q 13.33$ & 4 & Chr19:49713645-49714045 & $-0.03(-0.05 ;-0.02)$ & $1.1 \mathrm{e}-03$ \\
\hline
\end{tabular}

The italics boxes indicate DMRs that are common to more than one exposure 
Table 3 Table of replication in Generation R of the significant single CpG sites found in ALSPAC

\begin{tabular}{|c|c|c|c|c|c|c|}
\hline \multirow[t]{2}{*}{ Probe ID } & \multicolumn{3}{|c|}{$\begin{array}{l}\text { ALSPAC mid-pregnancy } \\
\text { depression }\end{array}$} & \multicolumn{3}{|c|}{ Generation R Study } \\
\hline & Beta & SE & $p$-value & Beta & SE & $p$-value \\
\hline cg08667740 & -0.025 & 0.005 & 3.90E-08 & 0.003 & 0.002 & 0.186 \\
\hline cg22868225 & -0.005 & 0.001 & $5.89 \mathrm{E}-08$ & -0.001 & 0.001 & 0.672 \\
\hline
\end{tabular}

DNA methylation at seven DMRs, but no individual CpG sites in our main analysis in ALSPAC.

Depression in mid-pregnancy, late pregnancy, and at both time points (throughout pregnancy) was associated with 7, 23, and 2 DMRs, respectively. Depression in midpregnancy was the only exposure definition associated with any individual CpG sites with genome-wide significance. However, findings for the two identified CpGs (cg08667740 and cg22868225) did not replicate in Generation R. Although we found that $72 \% \mathrm{CpG}$ sites within DMRs identified in ALSPAC showed the same direction of association between antenatal maternal depression and offspring DNA methylation in Generation R, none of our findings replicated with statistical significance.

Although none of the ALSPAC DMRs replicated in the Generation $\mathrm{R}$ study with statistical significance, the direction of association was the same at some regions that have previously been associated with neurodevelopmental and psychiatric outcomes. Out of the $49 \mathrm{CpG}$ sites that had the same direction of association in both ALSPAC and the Generation R study 35 were hypomethylated in both cohorts and located in the promoter region of the HOXA5 gene. This gene has been associated with depression in early pregnancy and this gene family is known to have important roles in embryonic development $^{42}$ and has been seen to be downregulated in the prefrontal cortex of depressed suicide subjects ${ }^{43}$.

Another seven $\mathrm{CpG}$ sites that were all hypermethylated in both ALSPAC and the Generation R study mapped to the promoter region of the LYNX1 gene. This gene was previously found to be hypermethylated in the hippocampus from patients with major depressive disorders ${ }^{44}$. In this study they conducted both a genome-wide association study using the $450 \mathrm{~K}$ array and investigated the LYNX1 gene using bisulfite pyrosequencing. Both methods yielded the same result.

In the cord blood of the children exposed to depression at any time point in pregnancy we found a DMR spanning more than $20 \mathrm{CpG}$ sites mapping to the promotor region of the NNAT gene which is an imprinted gene that may be involved in brain development and in forming and maintaining the structure of the nervous system ${ }^{45}$.
The NNAT gene has been investigated previously in relation to maternal depression during pregnancy ${ }^{46,47}$. These studies investigated imprinted genes but none of them found any association between depression during pregnancy and changes in DNA methylation in cord blood of newborn infants in this gene.

In candidate gene studies, Devlin et al. ${ }^{13}$ and Oberlander et al. ${ }^{14}$ found associations between maternal depressed mood during pregnancy and variation in cord blood DNA methylation at the $S L C 6 A 4$ and $N R 3 C 1$ genes, respectively. These findings were not replicated in our study.

There has been one previous EWAS of maternal depressed mood or anxiety during pregnancy and cord blood methylation in just 13 children $^{48}$. Of the $10 \mathrm{CpG}$ sites differentially methylated with $\mathrm{FDR}<0.05$ in this study, $5 \mathrm{CpG}$ sites were associated with depression in the same direction in our study, but the smallest FDRcorrected $p$-value for these associations was 0.999 (Table S4).

\section{Strengths and limitations}

The ALSPAC study is a large birth cohort with very comprehensive, prospectively collected data on a population level reducing the possibility of recall bias or reverse causality.

Another strength to this study is the use of the EPDS, which is a self-reported screening tool for depression that correlates with clinical depression with a high sensitivity at the chosen cut off of 12 points ${ }^{19}$. However, it should be noted that this is a screening tool and therefore the prevalence of depression (20\%) in our study was higher than estimates for clinical diagnoses $(\sim 13 \%)^{1}$. It is interesting that we find at least some association between depression and offspring methylation in our study, given that women in our study are likely to have with milder forms of depression. In future studies it would be interesting to investigate the association between exposure to clinically verified depression and changes in DNA methylation in cord blood from the exposed infants.

A limitation to this study is the coverage of the $450 \mathrm{~K}$ BeadChip. It covers less than $2 \%$ of the CpG sites in the human epigenome. This limited coverage could result in an underestimation of the true methylation differences in epigenomic areas not covered by this array, including methylation variable loci that have previously been implicated in depression.

Even though, to our knowledge, this study is the largest on depression during pregnancy and cord blood methylation, inability to detect true associations in our discovery (ALSPAC) and replication (The Generation R Study) cohorts due to lack of statistical power is entirely possible. Furthermore, the Generation R Study used a different measure of depression to the ALSPAC cohort. The 
correlation between the two different mood scores used by ALSPAC (EPDS) and The Generation R Study (BSI) has been studied sparsely ${ }^{49}$ and the relatively low may correlation contribute to the lack of replication. Using two different instruments may however be considered a strength in this study. The convergent direction of associations between the cohorts point towards an effect of depression rather than an instrument specific effect.

The methylation data used in this study are derived from cord blood, and it could be argued that it would be more appropriate to study the association of maternal depression and DNA methylation in a more biologically relevant tissue, for example, infant brain tissue. However, aside from the obvious ethical and feasibility issues, there is increasing evidence to suggest that peripheral tissues may have some utility in epigenetic epidemiological studies of brain disorders ${ }^{50,51}$.

The composition of cells in umbilical cord blood may confound results if the composition is related to exposure or outcome. In this study, it is plausible that maternal depression, or exposures related to depression, such as smoking, could influence cell composition in cord blood. In order to limit the effect of this we adjusted for cord blood cell composition using the Andrews and Bakulski cord blood reference panel. We also investigated the association between exposure and estimated cell proportion. Maternal depression was not associated with the estimated cell proportions (Table S5).

We aknowledge, that it could be a limitation to this study, that we have not confirmed the significant CpG sites by more quantitative analyses, e.g. pyrosequencing. The $450 \mathrm{~K}$ array is however well validated, and yields values consistent with pyrosequencing ${ }^{52}$.

We have chosen the included covariates using the theory of DAGs and have therefore not included covariates often included in previous studies such as, e.g. child-sex and gestational age that are not true confounders with direct effect on both exposure and outcome. Conditioning on such covariates could confound the results and either over- or underestimate the true association depending on the source of bias $^{53,54}$.

The possibility of residual confounding cannot be ruled out but the sample size was not sufficient for the addition of more variables to the analysis.

However, despite this, to our knowledge, being the largest study of maternal depression and cord blood methylation to date, the sample size was not sufficient to allow for more stratified analyses or the addition of more variables to the multivariable analyses.

More smokers were found among depressed women compared to controls. Thus, residual confounding by smoking may exist. However, we found no overlap between the top CpG sites found in our analyses of depressed women vs. controls and the smoking-associated
CpG sites from a previous study ${ }^{55}$. This suggests that smoking may not be a strong confounder in our study.

Finally, the effect sizes in this study are rather small. The biological significance of this is unclear, and could not be explored further due to the lack of data such as downstream gene expression. Other environmental epigenetic studies do show that often only small-magnitude effects results from these exposures ${ }^{56}$, and future studies should evaluate the significance of these.

\section{Conclusion}

We identified some single and regional DNA methylation differences in umbilical cord blood of newborns exposed to maternal depression during pregnancy, compared to newborns that were unexposed but these results did not replicate in an independent cohort. Further studies with larger sample sizes and better epigenomic coverage investigating more tissue types are needed to further investigate whether epigenetics could be part of a biological pathway linking maternal depression during pregnancy to childhood outcomes.

\section{Acknowledgements}

We are extremely grateful to all the families who took part in this study, the midwives for their help in recruiting them, and the whole ALSPAC team, which includes interviewers, computer and laboratory technicians, clerical workers, research scientists, volunteers, managers, receptionists, and nurses. The UK Medical Research Council and Wellcome (grant ref: 102215/2/13/2) and the University of Bristol provide core support for ALSPAC. This study was also supported by the NIHR Biomedical Research Centre at the University Hospitals Bristol NHS Foundation Trust and the University of Bristol. The views expressed in this publication are those of the author(s) and not necessarily those of the NHS, the National Institute for Health Research, or the Department of Health. ARIES was funded by the BBSRC (BBI025751/1 and BB/I025263/1). ARIES is maintained under the auspices of the MRC Integrative Epidemiology Unit at the University of Bristol (MC_UU_12013/2 and MC_UU_12013/8). A.N. and H.T. are supported by a grant of the Dutch Ministry of Education, Culture, and Science and the Netherlands Organization for Scientific Research (NWO grant No. 024.001.003, Consortium on Individual Development). The work of H.T. is further supported by a European Union's Horizon 2020 research and innovation program (Contract grant number: 633595, DynaHealth) and a NWO-VICl grant (NWO-ZonMW: 016.VICl.170.200). The Generation R Study is conducted by the Erasmus MC, University Medical Center Rotterdam in close collaboration with the School of Law and Faculty of Social Sciences of the Erasmus University Rotterdam, the Municipal Health Service Rotterdam area, Rotterdam, the Rotterdam Homecare Foundation, Rotterdam and the Stichting Trombosedienst \& Artsenlaboratorium Rijnmond (STAR-MDC), Rotterdam. We gratefully acknowledge the contribution of children and parents, general practitioners, hospitals, midwives, and pharmacies in Rotterdam. The study protocol was approved by the Medical Ethical Committee of Erasmus MC, Rotterdam. Written informed consent was obtained for all participants. The generation and management of the Illumina 450K methylation array data (EWAS data) for the Generation R Study was executed by the Human Genotyping Facility of the Genetic Laboratory of the Department of Internal Medicine, Erasmus MC, the Netherlands. We thank Ms. Sarah Higgins, Ms. Mila Jhamai, Dr. Marjolein Peters, Dr. Lisette Stolk, Mr. Michael Verbiest, Mr. Marijn Verkerk, and Dr. Alexander Teumer for their help in creating the EWAS

database and the analysis pipeline. The Generation R Study is made possible by financial support from the Erasmus Medical Center, Rotterdam, the Erasmus University Rotterdam, the Netherlands Organization for Health Research and Development and the Dutch Ministry of Health, Welfare and Sport. The EWAS data was funded by a grant to V.W.J. from the Netherlands Genomics Initiative (NGI)/Netherlands Organisation for Scientific Research (NWO) Netherlands Consortium for Healthy Aging (NCHA; project nr. 050-060-810), by funds from 
the Genetic Laboratory of the Department of Internal Medicine, Erasmus MC, and by a grant from the National Institute of Child and Human Development (R01HD068437). V.W.J. received a grant from the Netherlands Organization for Health Research and Development (VIDI 016.136.361) and a Consolidator Grant from the European Research Council (ERC-2014-CoG-648916). J.F.F. has received funding from the European Union's Horizon 2020 research and innovation programme under grant agreement No. 633595 (DynaHEALTH). This study received funding from the European Union's Horizon 2020 research and innovation programme (733206, LIFECYCLE).

\section{Author details}

${ }^{1}$ Perinatal Epidemiology Research Unit, Department of Clinical Medicine, Health, Aarhus University, Aarhus, Denmark. ${ }^{2} \mathrm{MRC}$ Integrative Epidemiology Unit, University of Bristol, Bristol, UK. ${ }^{3}$ School of Oral and Dental Sciences, University of Bristol, Bristol, UK. ${ }^{4}$ Population Health Sciences, Bristol Medical School, University of Bristol, Bristol, UK. ${ }^{5}$ Avon \& Wiltshire Partnership NHS Mental Health Trust, Bristol, UK. ${ }^{6}$ Department of Paediatrics, Aarhus University Hospital, Aarhus, Denmark. ${ }^{7}$ Department of Obstetrics and Gynaecology, Aarhus University Hospital, Aarhus, Denmark. ${ }^{8}$ The Lundbeck Foundation Initiative for Integrative Psychiatric Research, iPSYCH, Aarhus, Denmark. ${ }^{9}$ Translational Neuropsychiatric Unit, Aarhus University Hospital, Risskov, Denmark. ${ }^{10}$ Department of Child and Adolescent Psychiatry and Psychology, Erasmus MC, Rotterdam, The Netherlands. " Department of Epidemiology, Erasmus MC, Rotterdam, The Netherlands. ${ }^{12}$ The Generation R Study Group, Erasmus MC, Rotterdam, The Netherlands. ${ }^{13}$ Department of Pediatrics, Erasmus MC, Rotterdam, The Netherlands

\section{Conflict of interest}

The authors declare that they have no conflict of interest.

\section{Publisher's note}

Springer Nature remains neutral with regard to jurisdictional claims in published maps and institutional affiliations.

Supplementary Information accompanies this paper at (https://doi.org/ 10.1038/s41398-018-0286-4).

Received: 23 September 2017 Revised: 26 August 2018 Accepted: 10 September 2018

Published online: 07 November 2018

\section{References}

1. Gavin, N. I. et al. Perinatal depression: a systematic review of prevalence and incidence. Obstet. Gynecol. 106(Pt 1), 1071-1083 (2005).

2. Vigod, S. N., Wilson, C. A. \& Howard, L. M. Depression in pregnancy. BMJ (Clin. Res. Ed.) 352, i1547 (2016).

3. Stein, A. et al. Effects of perinatal mental disorders on the fetus and child. Lancet 384, 1800-1819 (2014).

4. Rai, D. et al. Parental depression, maternal antidepressant use during pregnancy, and risk of autism spectrum disorders: population based case-control study. BMJ 346, f2059 (2013).

5. Rai, D. et al. Antidepressants during pregnancy and autism in offspring: population based cohort study. BMJ (Clin. Res. Ed.) 358, j2811 (2017).

6. Hay, D. F., Pawlby, S., Waters, C. S., Perra, O. \& Sharp, D. Mothers' antenatal depression and their children's antisocial outcomes. Child Dev. 81, 149-165 (2010).

7. Lewis, A. J., Austin, E., Knapp, R., Vaiano, T. \& Galbally, M. Perinatal maternal mental health, fetal programming and child development. Healthcare (Basel, Switzerland) 3, 1212-1227 (2015).

8. Malm, H. et al. Gestational exposure to selective serotonin reuptake inhibitors and offspring psychiatric disorders: a national register-based study. J. Am. Acad. Child Adolesc. Psychiatry 55, 359-366 (2016).

9. Van den Bergh, B. R. Developmental programming of early brain and behaviour development and mental health: a conceptual framework. Dev. Med. Child Neurol. 53(Suppl 4), 19-23 (2011).
10. Monk, C., Spicer, J. \& Champagne, F. A. Linking prenatal maternal adversity to developmental outcomes in infants: the role of epigenetic pathways. Dev. Psychopathol. 24, 1361-1376 (2012).

11. Kinney, D. K., Miller, A. M., Crowley, D. J., Huang, E. \& Gerber, E. Autism prevalence following prenatal exposure to hurricanes and tropical storms in Louisiana. J. Autism Dev. Disord. 38, 481-488 (2008).

12. Rai, D. et al. Prenatal and early life exposure to stressful life events and risk of autism spectrum disorders: population-based studies in Sweden and England. PLOS ONE 7, e38893 (2012).

13. Devlin, A. M., Brain, U., Austin, J. \& Oberlander, T. F. Prenatal exposure to maternal depressed mood and the MTHFR C677T variant affect SLC6A4 methylation in infants at birth. PLOS ONE 5, e12201 (2010).

14. Oberlander, T. F. et al. Prenatal exposure to maternal depression, neonatal methylation of human glucocorticoid receptor gene (NR3C1) and infant cortisol stress responses. Epigenetics 3, 97-106 (2008).

15. Boyd, A. et al. Cohort Profile: the 'children of the 90s' - the index offspring of the Avon Longitudinal Study of Parents and Children. Int. J. Epidemiol. 42, 111-127 (2013).

16. Headley, J., Northstone, K., Simmons, H. \& Golding, J. Medication use during pregnancy: data from the Avon Longitudinal Study of Parents and Children. Eur. J. Clin. Pharmacol. 60, 355-361 (2004).

17. Avon Longitudianl Study of Parents and Children. http://www.bristol.ac.uk/ alspac/researchers/access/.

18. Cox, J. L., Holden, J. M. \& Sagovsky, R. Detection of postnatal depression. Development of the 10-item Edinburgh Postnatal Depression Scale. Br. J. Psychiatry 150, 782-786 (1987).

19. Kozinszky, Z. \& Dudas, R. B. Validation studies of the Edinburgh Postnatal Depression Scale for the antenatal period. J. Affect. Disord. 176, 95-105 (2015).

20. Pearson, R. M. et al. Maternal depression during pregnancy and the postnatal period: risks and possible mechanisms for offspring depression at age 18 years. JAMA Psychiatry 70, 1312-1319 (2013).

21. Sharp, G. C. et al. Maternal pre-pregnancy BMl and gestational weight gain, offspring DNA methylation and later offspring adiposity: findings from the Avon Longitudinal Study of Parents and Children. Int. J. Epidemiol. 44, 1288-1304 (2015).

22. Relton, C. L. et al. Data Resource Profile: Accessible Resource for Integrated Epigenomic Studies (ARIES). Int. J. Epidemiol. 44, 1181-1190 (2015).

23. Bibikova, M. et al. High density DNA methylation array with single CpG site resolution. Genomics 98, 288-295 (2011).

24. Pidsley, R. et al. A data-driven approach to preprocessing Illumina 450K methylation array data. BMC Genomics 14, 293 (2013).

25. Efficient algorithms for analyzing DNA methylation data: meffil. https://github. com/perishky/meffil.

26. Tukey J. W. Exploratory Data Analysis (1977).

27. Greenland, S., Pearl, J. \& Robins, J. M. Causal diagrams for epidemiologic research. Epidemiology 10, 37-48 (1999).

28. Houseman, E. A. et al. DNA methylation arrays as surrogate measures of cell mixture distribution. BMC Bioinformatics 13, 86 (2012).

29. Bakulski, K. M. et al. DNA methylation of cord blood cell types: applications for mixed cell birth studies. Epigenetics 11, 354-362 (2016).

30. Leek, J. T. \& Storey, J. D. Capturing heterogeneity in gene expression studies by surrogate variable analysis. PLoS Genet. 3, 1724-1735 (2007).

31. Rakyan, V. K., Down, T. A., Balding, D. J. \& Beck, S. Epigenome-wide association studies for common human diseases. Nat. Rev. Genet. 12, 529-541 (2011).

32. Pedersen, B. S., Schwartz, D. A., Yang, I. V. \& Kechris, K. J. Comb-p: software for combining, analyzing, grouping and correcting spatially correlated P-values. Bioinformatics 28, 2986-2988 (2012).

33. Šidák, Z. Rectangular confidence regions for the means of multivariate normal distributions. J. Am. Stat. Assoc. 62, 626-633 (1967).

34. Phipson, B., Maksimovic, J. \& Oshlack, A. missMethyl: an R package for analyzing data from Illumina's HumanMethylation450 platform. Bioinformatics 32, 286-288 (2016).

35. Ashburner, M. et al. Gene ontology: tool for the unification of biology. Nat Genet. 25, 25-29 (2000).

36. Kanehisa, M. \& Goto, S. KEGG: kyoto encyclopedia of genes and genomes. Nucleic Acids Res. 28, 27-30 (2000).

37. Kooijman, M. N. et al. The Generation R Study: design and cohort update 2017 Eur. J. Epidemiol. 31, 1243-1264 (2016).

38. Kruithof, C. J. et al. The Generation R Study: Biobank update 2015. Eur. J. Epidemiol. 29, 911-927 (2014). 
39. Derogatis, L. R. \& Melisaratos, N. The Brief Symptom Inventory: an introductory report. Psychol. Med. 13, 595-605 (1983).

40. Zhang, $\mathrm{H}$. et al. Identification of methylation quantitative trait loci (mQTLs) influencing promoter DNA methylation of alcohol dependence risk genes. Hum. Genet. 133, 1093-1104 (2014).

41. Gaunt, T. R. et al. Systematic identification of genetic influences on methylation across the human life course. Genome Biol. 17, 61 (2016).

42. Wang, H. D. et al. DNA methylation study of fetus genome through a genome-wide analysis. BMC Med. Genomics 7, 18 (2014).

43. Smalheiser, N. R. et al. MicroRNA expression is down-regulated and reorganized in prefrontal cortex of depressed suicide subjects. PLOS ONE 7, e33201 (2012).

44. Kaut, O. et al. Aberrant NMDA receptor DNA methylation detected by epigenome-wide analysis of hippocampus and prefrontal cortex in major depression. Eur. Arch. Psychiatry Clin. Neurosci. 265, 331-341 (2015).

45. Dou, D. \& Joseph, R. Structure and organization of the human neuronatin gene. Genomics 33, 292-297 (1996).

46. Liu, Y. et al. Depression in pregnancy, infant birth weight and DNA methylation of imprint regulatory elements. Epigenetics 7, 735-746 (2012).

47. Vidal, A. C. et al. Maternal stress, preterm birth, and DNA methylation at imprint regulatory sequences in humans. Genet. Epigenet. 6, 37-44 (2014).

48. Non, A. L., Binder, A. M., Kubzansky, L. D. \& Michels, K. B. Genome-wide DNA methylation in neonates exposed to maternal depression, anxiety, or SSRI medication during pregnancy. Epigenetics 9, 964-972 (2014).
49. Orun, E., Yalcin, S. S. \& Mutlu, B. Relations of maternal psychopathologies, social-obstetrical factors and mother-infant bonding at 2-month postpartum: a sample of Turkish mothers. World J. Pediatr. 9, 350-355 (2013).

50. Davies, M. N. et al. Functional annotation of the human brain methylome identifies tissue-specific epigenetic variation across brain and blood. Genome Biol. 13, R43 (2012)

51. Clive, M. L. et al. Discovery and replication of a peripheral tissue DNA methylation biosignature to augment a suicide prediction model. Clin. Epigenet. 8, 113 (2016).

52. Roessler, J. et al. Quantitative cross-validation and content analysis of the 450k DNA methylation array from Illumina, Inc. BMC Res. Notes 5, 210 (2012).

53. Cole, S. R. et al. Illustrating bias due to conditioning on a collider. Int. J. Epidemiol. 39, 417-420 (2010).

54. Richiardi, L., Bellocco, R. \& Zugna, D. Mediation analysis in epidemiology: methods, interpretation and bias. Int. J. Epidemiol. 42, 1511-1519 (2013)

55. Joubert, B. R. et al. DNA methylation in Newborns and Maternal Smoking in Pregnancy: Genome-wide Consortium Meta-analysis. Am. J. Hum. Genet. 98, 680-696 (2016).

56. Breton, C. V. et al. Small-magnitude effect sizes in epigenetic end points are important in children's environmental health studies: The Children's Environmental Health and Disease Prevention Research Center's Epigenetics Working Group. Environ. Health Perspect. 125, 511-526 (2017). 